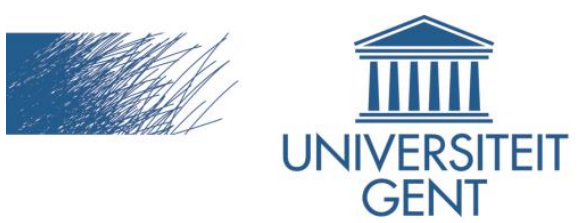

biblio.ugent.be

The UGent Institutional Repository is the electronic archiving and dissemination platform for all UGent research publications. Ghent University has implemented a mandate stipulating that all academic publications of UGent researchers should be deposited and archived in this repository. Except for items where current copyright restrictions apply, these papers are available in Open Access.

This item is the archived peer-reviewed author-version of:

Implementing a qualitative calculus to analyse moving point objects

Delafontaine Matthias, Cohn Anthony G., Van de Weghe Nico

In: Expert Systems With Applications, Vol. 38 (5), p. 5187-5196, 2011.

doi: 10.1016/j.eswa.2010.10.042

To refer to or to cite this work, please use the citation to the published version:

Delafontaine, M., Cohn, A. G. \& Van de Weghe, N. (2011) Implementing a qualitative calculus to analyse moving point objects. Expert Systems with Applications, 38 (5), p. 5187-5196. 


\title{
Implementing a qualitative calculus to analyse moving point objects
}

Delafontaine M., Cohn A. G., Van de Weghe N.

\begin{abstract}
Due to recent technological advances in position-aware devices, data about moving objects is becoming ubiquitous. Yet, it is a major challenge for spatial information systems to offer tools for the analysis of motion data, thereby evolving from static to dynamic frameworks. This chapter aims to contribute to this area by introducing an implementation prototype for an information system based on the Qualitative Trajectory Calculus, a spatiotemporal calculus to represent and reason about moving point objects.
\end{abstract}

Keywords. Qualitative calculus - Moving point objects - Implementation

\section{Introduction}

Capabilities to track individual moving objects have recently developed, along with the technological advances concerning position-aware devices, navigation systems, electronic transaction networks and surveillance systems (Laube et al. 2007). Nowadays, hi-tech devices such as mobile phones, digicams, GPS receivers and RFIDs, are omnipresent and allow for a low cost capture of high resolution trajectories ${ }^{1}$ of moving objects, whether these are human beings (Wang, Hu \& Tan 2003, Gau et al. 2004, Nielsen \& Hovgesen 2004, Michael et al. 2006), animals (DeCesare, Squires \& Kolbe 2005, Yasuda \& Arai 2005, Kritzler, Raubal \& Kruger 2007, Laube et al. 2007, Gagliardo et al. 2007), vehicles (Brunk \& Davis 2002, Brakatsoulas et al. 2005, Hvidberg 2006), or even projectiles (Grace 2000). As is generally recognized, this large potential of individually-based trajectory data heralds a new era of movement analysis (Eagle \& Pentland 2006, Laube et al. 2007) in order to feed a broad range of application fields from ethology over traffic management to sport scene analysis and weapon guidance.

In the past decade, GIScientists from multiple disciplines have created a sound theoretical basis regarding the modelling, representation, analysis and extraction of knowledge from motion data (see among others (Laube, Imfeld \& Weibel 2005, Güting, de Almeida \& Ding 2006, Giannotti \& Pedreschi 2008, Spaccapietra et al. 2008) for an overview).

Despite these considerable efforts, common analyses of trajectory data remained limited with respect to scope and sophistication (Laube et al. 2007), and much of this theoretical work is not well reflected in tools offered by current spatial information systems (Wentz, Campbell \& Houston 2003).

One of the research fields which until now has remained largely theoretical is the domain of qualitative reasoning (QR). However, one of the key motivations for QR lies in its applicability for user

\footnotetext{
${ }^{1}$ Although also denoted as geospatial lifelines (Mark 1998, Hornsby \& Egenhofer 2002, Laube, van Kreveld \& Imfeld 2005) others refer to trajectories (Gottfried 2008, Orlando et al. 2007, Brakatsoulas, Pfoser \& Tryfona 2004, Spaccapietra et al. 2008, Gudmundsson, van Kreveld \& Speckmann 2007), as we will do for consistency with the QTC calculus.
} 
interactive information systems, where qualitative information tallies much more with human intuition, communication and decision making than quantitative information (Egenhofer \& Mark 1995, Renz, Rauh \& Knauff 2000, Monferrer \& Lobo 2002). In the past, several qualitative spatial and temporal calculi have been introduced, first and foremost as a reasoning tool: the Interval Algebra (Allen 1983), the Point Algebra (Vilain \& Kautz 1986), the Cardinal Direction Calculus (Frank 1991), the Doublecross Calculus (Freksa 1992b), the Region Connection Calculus (Randell, Cui \& Cohn 1992) and the Oriented Point Reasoning Algebra (Moratz, Dylla \& Frommberger 2005) to name but a few.

Yet, the usefulness of these calculi often remains questionable and needs a thorough evaluation in terms of suitability, relevance and scope of potential applications. Wallgrün et al. (2007) already made a general attempt in that direction with the development of a qualitative spatial reasoning toolbox SparQ to allow for an easy integration in applications. Another effort comes from El-Geresy and Abdelmoty (2004) with the introduction of a qualitative spatial reasoning engine SPARQS for the automatic derivation of composition tables. Another relevant line of work is that of Renz and $\mathrm{Li}$ (2008) who have largely automated the task of determining the maximal tractable fragments for qualitative calculi.

Whereas most of the above mentioned calculi either stick to spatial or temporal issues, just a few of them combine both to allow for spatiotemporal reasoning. One of them of particular interest to the domain of moving objects is the Qualitative Trajectory Calculus (QTC) (Van de Weghe 2004), which considers disjoint moving points objects (MPOs). We believe that QTC constitutes a basis to represent and reason about moving objects, and thus its implementation in an information system would provide a practical tool to support the analysis of moving objects.

This chapter introduces an implementation prototype for the Basic $\left(\mathrm{QTC}_{\mathrm{B}}\right)$ and Double-Cross $\left(\mathrm{QTC}_{\mathrm{C}}\right)$ calculi (Van de Weghe et al. 2006). Our aim is to show how QTC can be implemented in an information system in a generic way, to introduce a methodology for handling continuous data sampled at discrete times suitable for QTC, and to demonstrate the applicability of such a system. The remainder of this chapter is organised as follows. Section 2 sketches a brief overview of QTC and its different types, including an informal account of $\mathrm{QTC}_{\mathrm{B}}$ and $\mathrm{QTC}_{\mathrm{C}}$ (section 2). Section 3 introduces a conceptual modal and a prototype QTC-based information system. In section 4, the use of this system is illustrated in two different case studies. Section 5 presents a detailed discussion, and finally, section 6 draws some conclusions and considers possible future work.

\section{The Qualitative Trajectory Calculus (QTC)}

\subsection{Types of QTC}

QTC was introduced by Van de Weghe (2004) as a qualitative calculus to represent and reason about moving objects. The QTC formalism defines relations between a pair of disjoint MPOs. These MPOs are assumed to evolve continuously in space and time. Due to the consideration of different spaces and frames of reference, the following types of QTC have been elaborated (Van de Weghe 2004):

- Basic type - QTC

- Double-Cross type - $\mathrm{QTC}_{\mathrm{C}}$

- Network type- QTC $_{N}$ 
- Shape type-QTCS

QTC $_{B}$ (Basic) and QTC $_{C}$ (Double-Cross) both deal with MPOs having a free trajectory in an $n$ dimensional space. In $\mathrm{QTC}_{\mathrm{B}}$, relations are determined referring to the Euclidian distance between two MPOs (Figure 1a) (Van de Weghe et al. 2006), while QTC $_{C}$ relations use the double cross between them (Figure 1b) (Van de Weghe et al. 2005a), as introduced by Zimmerman and Freksa (1996).

QTC $_{N}$ (Network) (Bogaert 2008, Delafontaine et al. 2008) focuses on the special case of MPOs which trajectories are constrained by a network, such as cars in a city. Since both the Euclidean distance and the double cross concepts ignore the spatial configuration of a potential underlying network, they are not well suited for $\mathrm{QTC}_{\mathrm{N}}$. Therefore, $\mathrm{QTC} \mathrm{N}_{\mathrm{N}}$ relations rely on the shortest paths in the network between the considered MPOs (Figure 1c).

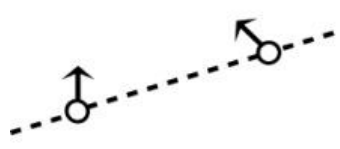

(a)

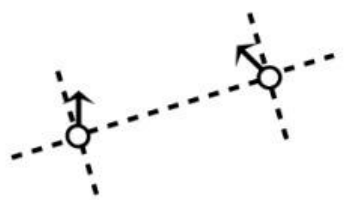

(b)

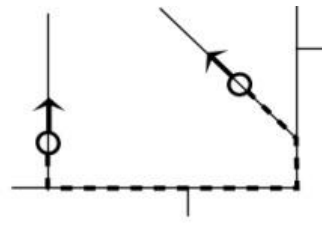

(c)

Figure 1 - Two MPOs represented in a typical $\operatorname{QTC}_{B}(a), \operatorname{QTC}_{C}(b)$, and $\mathrm{QTC}_{N}(c)$ setting. The frame of spatial reference is represented by the dashed line.

Finally, QTC s (Shape) is a calculus to represent and compare trajectory shapes, completely abstracting from the actual MPOs (Van de Weghe et al. 2005b).

In QTC, space and time (and thus the motion of MPOs) are assumed to be continuous. Therefore, QTC relations may change over time according to the laws of continuity. In what follows, we will use the term transition to denote the continuous change of one relation into a conceptual neighbouring relation (Freksa 1992a), thus without passing intermediate relations. Each transition occurs at an instant, i.e. point in time, which we will term transition instant.

All QTC calculi are associated with a set of jointly exhaustive and pairwise disjoint (JEPD) base relations. Consequently, there is one and only one relation for each pair of coexisting MPOs at each time instant. In addition, due to continuity, the concurrent movement of two MPOs over a time interval is uniquely mapped to a sequence of conceptual neighbouring base relations.

\subsection{Unconstrained movement}

Both QTC $_{B}$ and QTC $_{C}$ were developed to represent and reason about MPO movements in a free Euclidean space. Van de Weghe et al. (2006, 2005c) introduced four types (B11, B12, B21, B22) of QTC $_{B}$, and two types $(C 21, C 22)$ of QTC $_{C}$, although more subtypes could be defined on the same basis. All relations in each of these six types are composed of multiple relation symbols, each of which has the three-valued qualitative domain $\{-, 0,+\}$. These symbols rely on (a subset of) the following relationships:

For a pair of MPOs $k$ and $l$, and a time instant $t$ (Figure 2):

$k^{t}$ denotes the point location of $k$ at $t$ 
$\overrightarrow{v_{k}^{t}}$ denotes the velocity vector of $k$ at $t$

$k^{t} l^{t}$ denotes the straight line between $k^{t}$ and $l^{t}$

$\alpha^{t}$ denotes the positive angle $\left(0^{\circ} \leq \alpha^{t}<360^{\circ}\right)$ between $\overrightarrow{k^{t} l^{t}}$ and $\overrightarrow{v_{k}^{t}}$

$\beta^{t}$ denotes the positive angle $\left(0^{\circ} \leq \beta^{t}<360^{\circ}\right)$ between $\overrightarrow{l^{t} k^{t}}$ and $\overrightarrow{v_{l}^{t}}$

$\gamma^{t}$ denotes the minimum absolute angle $\left(0^{\circ} \leq \gamma^{t} \leq 90^{\circ}\right)$ between $k^{t} l^{t}$ and $\overrightarrow{v_{k}^{t}}$

$\delta^{t}$ denotes the minimum absolute angle $\left(0^{\circ} \leq \delta^{t} \leq 90^{\circ}\right)$ between $k^{t} l^{t}$ and $\overrightarrow{v_{l}^{t}}$

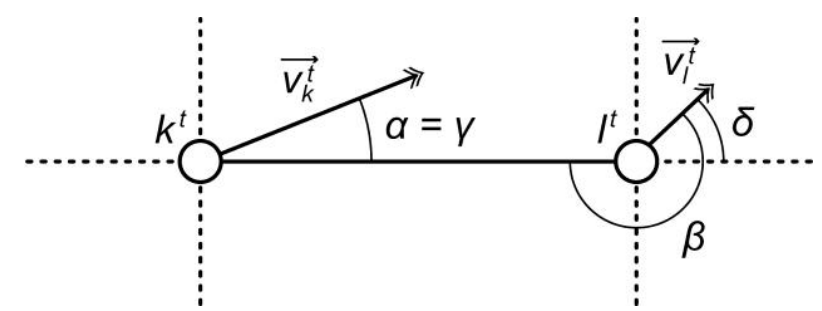

Figure 2 - Properties of two MPOs $k$ and / at a time instant $t$.

Then at $t$ :

A. $-: k$ is moving towards $l$

$\alpha^{t}<90^{\circ} \vee \alpha^{t}>270^{\circ}$

$+: k$ is moving away from $l$

$\alpha^{t}>90^{\circ} \wedge \alpha^{t}<270^{\circ}$

0 : all other cases ( $k$ is stable with respect to $l$ )

B. $-: l$ is moving towards $k$

$\beta^{t}<90^{\circ} \vee \beta^{t}>270^{\circ}$

$+: l$ is moving away from $k$

$\beta^{t}>90^{\circ} \wedge \beta^{t}<270^{\circ}$

0 : all other cases ( $l$ is stable with respect to $k$ )

C. $-: k$ is moving to the left of $\overrightarrow{k l}$

$$
\alpha^{t}>0^{\circ} \wedge \alpha^{t}<180^{\circ}
$$

$+: k$ is moving to the right of $\overrightarrow{k l}$

$\alpha^{t}>180^{\circ} \wedge \alpha^{t}<360^{\circ}$

0 : all other cases ( $k$ is stable with respect to $\overrightarrow{k l}$ )

D. $-: l$ is moving to the left of $\overrightarrow{l k}$

$$
\beta^{t}>0^{\circ} \wedge \beta^{t}<180^{\circ}
$$

$+: l$ is moving to the right of $\overrightarrow{l k}$

$$
\beta^{t}>180^{\circ} \wedge \beta^{t}<360^{\circ}
$$

0 : all other cases $(l$ is stable with respect to $\overrightarrow{l k}$ )

E. $-: k$ is moving faster than $l$

$\left|\overrightarrow{v_{k}^{t}}\right|>\left|\overrightarrow{v_{l}^{t}}\right|$

$+: k$ is moving slower than $l$

$$
\left|\overrightarrow{v_{k}^{t}}\right|<\left|\overrightarrow{v_{l}^{t}}\right|
$$

0 : all other cases ( $k$ is moving equally fast as $l$ )

F. $\quad-: k$ is moving at a smaller angle with respect to $k l$ than $l$ $\gamma^{t}<\delta^{t}$ 
$+: k$ is moving at a bigger angle with respect to $k l$ than $l$

$\gamma^{t}>\delta^{t}$

$0:$ all other cases ( $k$ and $l$ are moving at the same angle with respect to $k l$ )

Thus, the assessment of these six relation symbols requires knowledge on the instantaneous location, and velocity (i.e. speed and motion azimuth) of both MPOs. Table 1 presents the syntax of relations for all QTC $C_{B}$ and QTC $_{C}$ types, according to the above mentioned relationships A-F. Note that B11 and B12 have the same syntax as respectively B21 and B22: they differ in the number of spatial dimensions taken into account. According to these rules, a configuration where at time $t$ a zebra $z$ is moving away from a lion $l$ which in turn is approaching and catching up with the zebra can be described in B22 by ' + ' for A ( $z$ away from $l)$, ' - ' for B $(l$ towards $z)$, and ' - ' for $E(z$ slower than $l$ ), which we will write as $R_{\mathrm{B} 22}(z, l)=(+--)_{\mathrm{B} 22}$.

From an application point of view (cf. the lion and the zebra), the order of objects in the relation often does not matter. Hence, converse relations have to be taken into account. Converse relations in $\mathrm{QTC}_{B}$ and $\mathrm{OTC}_{C}$ can be obtained by interchanging the relation symbols $A$ with $B, C$ with $D$, and by replacing $E$ and $F$ with their inverse symbols. The inverse symbol for ' - ' is ' + ', for ' 0 ' is ' 0 ', and for ' + ' is ' - '. In the above example, the converse of $R_{\mathrm{B} 22}(z, l)$ would be $R_{\mathrm{B} 22}(l, z)=(-++)_{\mathrm{B} 22}$.

\begin{tabular}{ll}
\hline QTC type & Relation syntax \\
\hline B11 & (A B) \\
B12 & (A B E) \\
B21 & (A B) \\
B22 & (A B E) \\
C21 & (A B C D) \\
C22 & (A B C D E F) \\
\hline
\end{tabular}

Table 1 - Relation syntax for $\mathrm{QTC}_{\mathrm{B}}$ and $\mathrm{QTC}_{\mathrm{C}}$ subtypes.

\section{A QTC-based information system}

\subsection{Trajectory representations}

As QTC assumes spatial and temporal continuity, the location, the speed, and the motion azimuth of an MPO are assumed to be continuous functions of time. Hence, an MPO trajectory is a continuous set of points in space and time, which corresponds to the conventional mathematical notion of a curve at the spatial level, and to a simple closed interval at the temporal level.

Yet, in order to implement QTC in an information system, we need to consider how information systems, and GISs in particular, store and represent MPO trajectories. Longley et al. (2005, p. 70) argue that any representation is discrete, stating that "the world is infinitely complex, but computer systems are finite". To date, by far the most common way to store a trajectory, is as a set of spatial locations at $n$ consecutive time steps (Orlando et al. 2007, Turchin 1998, Yu et al. 2004, Yu \& Kim 2006, Gudmundsson, van Kreveld \& Speckmann 2007) which we will term fixes, according to Laube et al. (2005). Obviously, such a discrete set of fixes conflicts with the assumption of spatial and temporal continuity underlying QTC. Hence, fixes need to be interpolated in space and time to obtain continuous trajectories. 


\subsection{Conceptual model}

In order to implement QTC in an information system, an object-oriented design is proposed, shown in Figure 3. Part of this model has been based on the MPO modelling domain after Laube et al. (2005) where trajectories are build of a set of fixes (see section 3.3).

The MPO class represents dimensionless moving objects, whose spatiotemporal properties are described by one or more trajectories (instances of Trajectory). Each Trajectory maintains a list of one or more fixes (instances of Fix), that describe locations in space (Point) and time (Instant). Each Trajectory has a timeSpan which equals the time interval between its first and last fix. In order to represent continuous trajectories, the Trajectory class may have its own detached functions to interpolate in between fixes. The MPO's location, speed and motion azimuth at a specific time instant are respectively returned by the getLocation, getSpeed, and getAzimuth methods (constraint: time parameter must be within timeSpan).

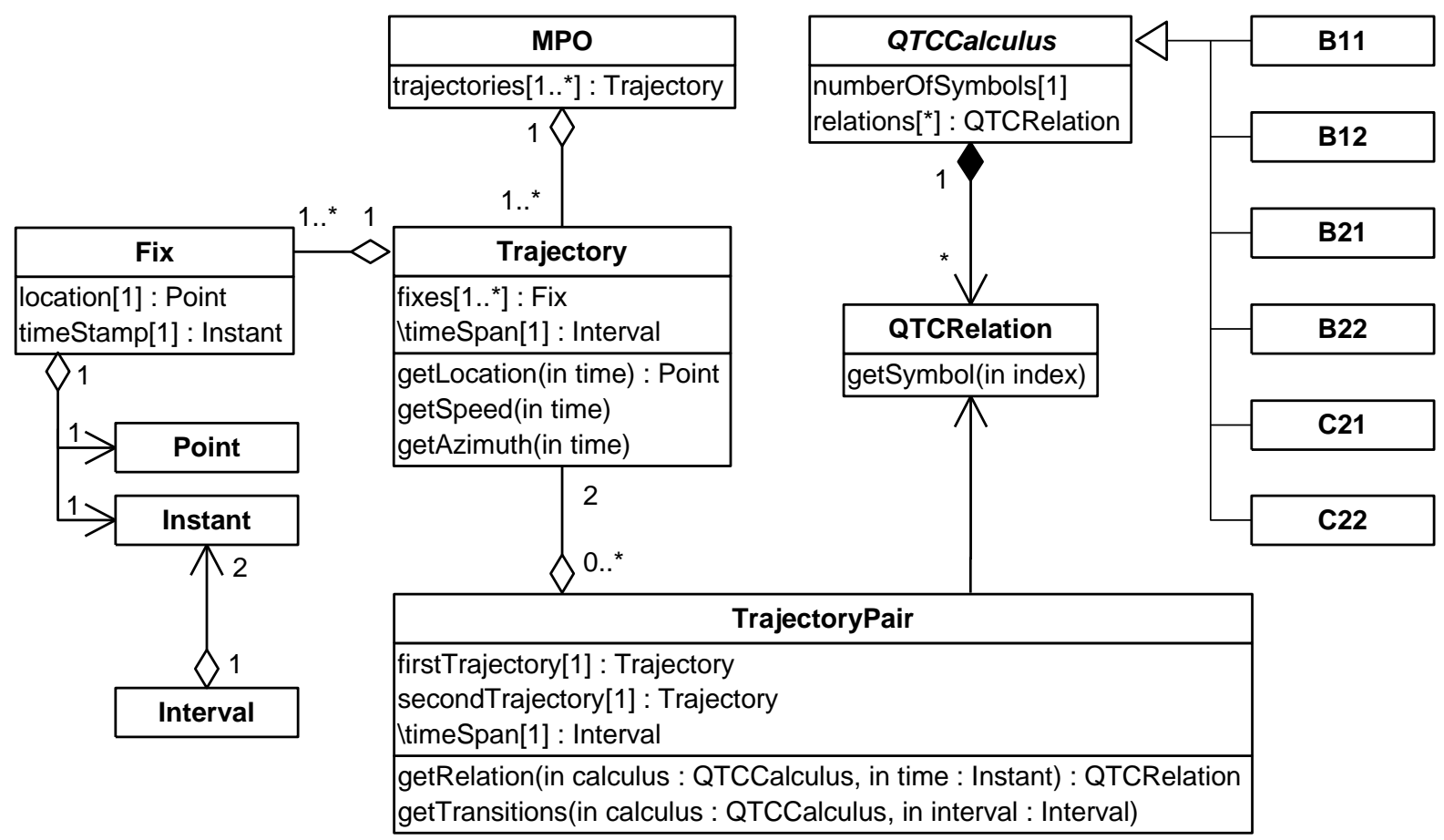

Figure 3 - UML class diagram for a QTC-based information system

As QTC applies to relations between two MPOs, a TrajectoryPair class is considered to embody an ordered pair of coexisting trajectories. The firstTrajectory and secondTrajectory properties refer to the respective Trajectory instances (constraints: firstTrajectory and secondTrajectory belong to a different MPO; firstTrajectory and secondTrajectory have an overlapping timeSpan). Each TrajectoryPair has a timeSpan which equals the temporal overlap between the timeSpan of firstTrajectory and seconTrajectory. Starting from the principles of continuity and JEPD (see section 2), we consider two basic operations that comprise the necessary conditions for an implementation of QTC:

- At each time instant there exists one and only one QTC relation between two MPOs (section 2.1). The getRelation method returns this relation for a given type of QTC at a given input time (constraint: the input time must be within the timeSpan of the TrajectoryPair). 
- Each TrajectoryPair can be associated with a chronologically ordered set of QTC relations and corresponding transition times (i.e. the instants at which the relations change) over a time interval during its timeSpan. This ordered mapping is returned by the getTransitions method for a given type of QTC (constraint: the input time interval must be during the timeSpan of the TrajectoryPair).

Specific types of QTC are modelled as subclasses of an abstract QTCCalculus class. They implement two properties: relations returns their set of base relations; numberOfSymbols returns the number of relation symbols in a relation.

QTC relations are represented by QTCRelation objects, which have a getSymbol method to return the individual relation symbol at the specified index (constraint: the index parameter must not exceed the numberOfSymbols of the QTCCalculus at hand).

\subsection{Implementation prototype}

Building on the conceptual model of section 3.2, we developed QTCAnalyst, a prototype QTC-based information system. In the remainder of this section, we give an overview of the assumptions and restrictions that underlie this implementation.

\section{Trajectories}

Although one can apply several methodologies to interpolate trajectory fixes in space and time, e.g. (Yu et al. 2004), QTCAnalyst relies on the following assumptions, being the most obvious and robust:

Assumption 1 - Trajectory polyline: In between two fix times, an MPO moves continuously along the straight line segment connecting both fixes.

Assumption 2 - Segment speed: In between two fixes, the speed of an MPO is constant.

Thus, a trajectory is represented as a polyline of which each vertex represents a fix, as shown in Figure 4. Though Assumptions 1 and 2 determine the trajectory of an MPO as a continuous function of time, they entail two discontinuities for MPOs at fixes: a discontinuity of motion azimuth due to Assumption 1 and a discontinuity of speed due to Assumption 2. Hence, contrary to getLocation, the getSpeed and getAzimuth methods are not defined for time instants that correspond to trajectory fixes of the MPO at hand. In order to get round this problem to determine the QTC relation at these instants, we will make use of a transition table, as discussed later in this section.

To enable an objective comparison of trajectories, QTCAnalyst assumes concurrency of fixes:

Assumption 3 - Concurrent observation: All trajectories are sampled at the same set of fix instants.

Whenever this assumption is not satisfied, fixes can always be resampled (interpolating as necessary) according to Assumptions 1 and 2 so that Assumption 3 is met. 


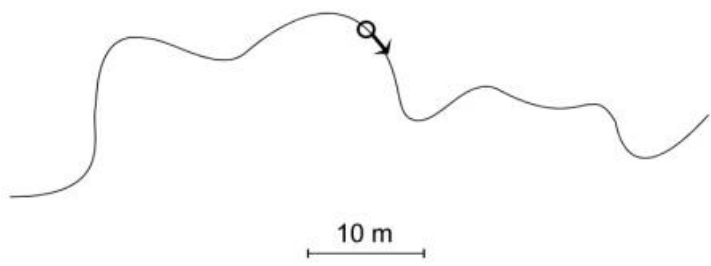

(a)

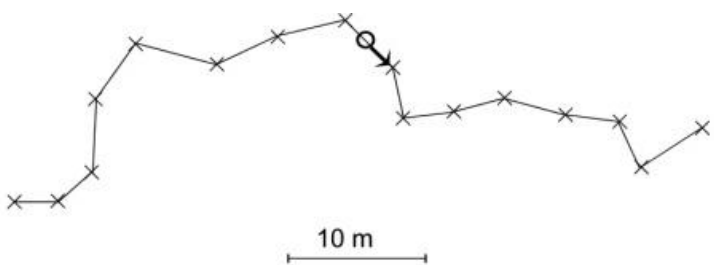

(b)

Figure 4 -A continuous MPO trajectory (a) and a representation of it according to Assumption 1 with fixes (crosses) per second (b).

\section{Relations}

As mentioned in section 3.2, an implementation of QTC requires a getRelation and a getTransition method. getRelation can be determined according to relationships A-F (section 2.2). However, this will be impossible for MPOs at fixes, since getSpeed and getAzimuth are ambiguous in that case due to Assumptions 1 and 2. In order to deduce relations for MPOs at fixes, QTCAnalyst relies on the laws of continuity, where Galton (2001) points out that ' - ' and ' + ' are 'dominated by' ' 0 ', from which follow these restrictions:

Restriction 1a - Intermediate ' 0 ': A transition from ' - ' to ' + ' must always pass the intermediate value ' 0 '.

Restriction $\mathbf{1 b}$ - Intermediate ' 0 ': A transition from ' + ' to ' - ' must always pass the intermediate value '0'.

Restriction 2a - Dominated '-': A '-' lasts over an open time interval.

Restriction 2b-Dominated ' + ': $A$ ' + ' lasts over an open time interval.

Restriction 2c-Dominant ' 0 ': $A$ ' 0 ' lasts over either a closed time interval, or a time instant.

Restriction 3a - Intermediate interval: There is always a closed time interval in between two '-' relations.

Restriction 3b - Intermediate interval: There is always a closed time interval in between two ' + ' relations.

There is always an adjacent open time interval before and after a time instant. For an instant $t$, let us denote these intervals respectively $t^{-}$and $t^{+}$. Then at transition instant $t$, due restrictions 1-3, we obtain the relation symbols presented in Table 2 . The implementation of getRelation at $t$ in QTCAnalyst is now as follows:

- For $t$ not at a fix time: getRelation uses relationships A - F (equations 1-12).

- For $t$ at a fix time: getRelation first computates the before and after relationships (A - F) using the locations at $t$ together with the speed and motion azimuth at respectively the preceding and following segment. Next, the transition table is employed to return the relation at $t$. 


\begin{tabular}{cc|ccc}
\multicolumn{2}{r|}{ Relation symbol at $t$} & \multicolumn{4}{|c}{ Relation symbol during $t^{+}$} \\
& & - & 0 & + \\
\hline Relation symbol & - & - & 0 & 0 \\
during $t^{-}$ & 0 & 0 & 0 & 0 \\
& + & 0 & 0 & +
\end{tabular}

Table 2 - Transition table for QTC relation symbols at transition instant $t$

\section{Transitions}

The getTransitions method returns the chronological sequence of QTC relations and the corresponding transition instants over a given valid time interval for the TrajectoryPair at hand. Due to dominance theory (Galton 2001), each transition instant corresponds to a ' 0 ' relationship symbol. By consequence, it suffices to assess at which instants a relationship symbol changes to or from ' 0 '. Due to Table 2, zero to two transitions might occur for each relationship symbol at one time instant (e.g. at fix times).

For time instants in between consecutive fixes, relationship A will be ' 0 ' when:

- $k$ is stationary (intra-object coinciding fixes, no transitions);

- $k$ and $l$ coincide. According to Assumptions 1-3, this situation occurs either at one intermediate time instant (collision, two transitions), or over the complete segment (inter-object coinciding fixes, no transitions);

- $\quad k^{t} l^{t}$ is perpendicular to $\overrightarrow{v_{k}^{t}}$ (equations 5.1-5.2). Due to Assumptions 1-3, this situation occurs either at one intermediate time instant (two transitions), or over the complete segment (no transitions).

The cases with two transitions (one from ' - ' or ' + ' to ' 0 ', the other one from ' 0 ' to respectively ' + ' or '-') are mutually exclusive and can be solved analytically on the basis of equations such as in Appendix A. Analogous to relationship A, it can be shown that there will be at most two transitions for $B-D$, and four transitions for $F$ for time intervals in between two consecutive fixes. Obviously, no transitions can occur for $\mathrm{E}$ due to Assumption 2 (only at fix times).

Thus, a TrajectoryPair consisting of $n$ pairs of concurrent fixes, will in a worst case scenario have $2 n+(2+2+2+2+0+4)(n-1)=14 n-12$ transitions over its total timeSpan.

\section{Prototype application}

QTCAnalyst was implemented in Visual Basic 6.5 using AutoCAD for visualisation and MS Excel for data input and output. Through a GUI, trajectories that answer Assumptions 1-3, can be loaded from fix data, and can be visualised in a conventional two-dimensional space (top view perspective), or in a space-time cube. TrajectoryPair instances can then be automatically generated for each canonical pair of coexisting Trajectory instances. Finally, the output of the getRelation and getTransitions methods can be exported, or visualised. In addition, QTCAnalyst is able to calculate and export simple summaries (see section 4.1), as well as relation patterns (see section 4.2), i.e. chains of subsequent relations, from the set of relations resulting from getTransitions. 


\section{Case studies}

In this section, we utilise QTCAnalyst to analyse QTC relations in two completely different contexts. The first case focuses on the $\mathrm{QTC}_{\mathrm{B}}$ relations between moving vehicles on a four lane one-way street. The second case deals with $\mathrm{QTC}_{C}$ relations of players in a squash contest.

\subsection{Cars on a street}

The study area is a straight section of about $130 \mathrm{~m}$ of a four lane one-way road in Ghent (Belgium), as schematized in Figure 5. This road is the south-north directed tail end of a highway exit for the centre of Ghent. Another single lane road converges with it immediately south of the study area, whereas there are traffic lights at the north end.

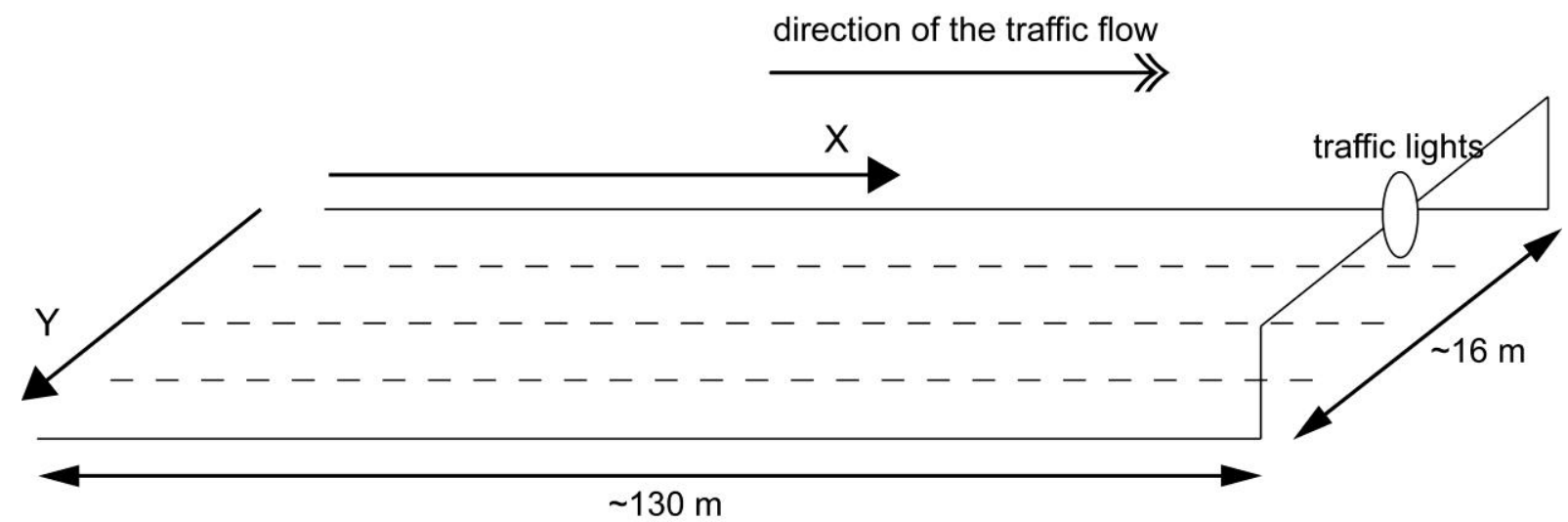

Figure 5 - Schematic sketch of the study area.

During the morning rush hour, a movie of the study area has been recorded with a steady camera from a high building in the neighbourhood for two minutes. This movie has been georeferenced to a local two-dimensional reference system in order to assess the relative positions of cars - treated as MPOs - on snapshots taken at a regular time step of $1 \mathrm{~s}$. The $x$-axis of this system is aligned with the road's centreline, whereas the $y$-axis is along the width dimension. The resolution in $y$ has intentionally been kept coarse, in order to eliminate insignificant shifts of cars that stay within their lane. Hence, we obtain a data set of 44 car trajectories with different time spans but with concurrent fixes (Assumption 3).

Of the 946 canonical trajectory pairs that exist for 44 trajectories, 503 have a temporal overlap, and hence enable the calculation of QTC relations. Table 3 and Table 4 list some of the QTCAnalyst results about the relations between these 503 valid pairs for the B21 and B22 calculi. The tables summarise the number of instantaneous occurrences, the number of occurrences over a time interval, the total number of occurrences, and the total duration for each relation aggregated over all valid pairs. 


\begin{tabular}{lrrrr}
\hline Relation & Instants & Intervals & Total & Duration (s) \\
\hline$(--)_{\mathrm{B} 21}$ & 0 & 1 & 1 & 0.2 \\
$(-+)_{\mathrm{B} 21}$ & 0 & 104 & 104 & 328.4 \\
$(-0)_{\mathrm{B} 21}$ & 3 & 3 & 6 & 3.0 \\
$(+-)_{\mathrm{B} 21}$ & 0 & 362 & 362 & 1581.7 \\
$(++)_{\mathrm{B} 21}$ & 0 & 4 & 4 & 0.7 \\
$(+0)_{\mathrm{B} 21}$ & 58 & 132 & 190 & 534.0 \\
$(0-)_{\mathrm{B} 21}$ & 146 & 344 & 490 & 2089.6 \\
$(0+)_{\mathrm{B} 21}$ & 49 & 172 & 221 & 738.4 \\
$(00)_{\mathrm{B} 21}$ & 567 & 204 & 771 & 3178.0 \\
Total & 823 & 1326 & 2149 & 8454.0 \\
\hline
\end{tabular}

Table 3 - Summary of QTC-B21 relations with their cumulative instant, interval, and total frequencies, and duration for 503 car pairs.

From Table 3, we may learn that all nine B21 base relations do have at least one occurrence. However, the occurrences are not equally distributed over this universe set. Since there is no significant natural order for cars in a street, we will focus our discussion on groups of converse relations. A first group is represented simply by the symmetric $\left(\begin{array}{ll}0 & 0\end{array}\right)_{\mathrm{B} 21}$ relation. It is the most common relation in the data set, lasting for almost $40 \%$ of the cumulative time. Perhaps this indicates the importance of collective stops (both cars standing still). A ' 0 ' relation symbol, however, does not imply object stationarity. For example, two cars driving next to each other in different

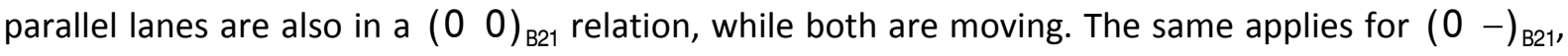
$(0+)_{\mathrm{B} 21}$, and their converse relations $(-0)_{\mathrm{B} 21}$, and $(+0)_{\mathrm{B} 21}$, which constitute another important group ( $40 \%$ of the total time). In these cases, at least one car is moving, whereas this is unknown for its associate. The last significant group consists of the relation $(-+)_{\mathrm{B} 21}$ and its converse $(+-)_{\mathrm{B} 21}$. They represent the regular situation of cars following one another. For the remaining two symmetric relations, the table indicates that situations where cars are converging $\left[(--)_{\mathrm{B} 21}\right]$ or diverging $[(+$ $\left.+)_{B 21}\right]$ are rare: they respectively occur once and four times, lasting for only fractions of seconds.

As shown in Table 4, only 21 of the 27 base relations occur in QTC-B22. The relative speed symbol of these relations can now be used in order to further refine the interpretations made for QTC-B21. Let us reconsider the groups of converse relations. For $\left(\begin{array}{ll}0 & 0\end{array}\right)_{\mathrm{B} 21}$, we can see that all intervals must be $\left(\begin{array}{lll}0 & 0 & 0\end{array}\right)_{\text {B22 }}$ since both share the same number of interval occurrences and have equal total duration. The remaining relations in this group, $\left(\begin{array}{lll}0 & 0 & -\end{array}\right)_{\mathrm{B} 22}$ and $\left(\begin{array}{lll}0 & 0 & +\end{array}\right)_{\mathrm{B} 22}$, only occur instantaneously. Due to the difference in relative speed, at least one object must be moving in this case. These are the typical transition relations of cars passing by each other. There number of occurrences gives a first indication of the number of overtake events in the dataset, although relation sequences would be needed for an exact assessment. 


\begin{tabular}{lrrrr}
\hline Relation & Instant & Interval & Total & Duration \\
\hline$(---)_{\mathrm{B} 22}$ & 0 & 1 & 1 & 0.2 \\
$(--+)_{\mathrm{B} 22}$ & 0 & 0 & 0 & 0.0 \\
$(--0)_{\mathrm{B} 22}$ & 0 & 0 & 0 & 0.0 \\
$(-+-)_{\mathrm{B} 22}$ & 0 & 110 & 110 & 306.5 \\
$(-++)_{\mathrm{B} 22}$ & 0 & 13 & 13 & 13.0 \\
$(-+0)_{\mathrm{B} 22}$ & 28 & 9 & 37 & 9.0 \\
$(-0-)_{\mathrm{B} 22}$ & 1 & 0 & 1 & 0.0 \\
$(-0+)_{\mathrm{B} 22}$ & 1 & 3 & 4 & 3.0 \\
$(-00)_{\mathrm{B} 22}$ & 1 & 0 & 1 & 0.0 \\
$(+--)_{\mathrm{B} 22}$ & 0 & 376 & 376 & 1197.7 \\
$(+-+)_{\mathrm{B} 22}$ & 0 & 157 & 157 & 290.1 \\
$(+-0)_{\mathrm{B} 22}$ & 250 & 79 & 329 & 94.0 \\
$(++-)_{\mathrm{B} 22}$ & 0 & 4 & 4 & 0.7 \\
$(+++)_{\mathrm{B} 22}$ & 0 & 0 & 0 & 0.0 \\
$(++0)_{\mathrm{B} 22}$ & 0 & 0 & 0 & 0.0 \\
$(+0-)_{\mathrm{B} 22}$ & 3 & 0 & 3 & 0.0 \\
$(+0+)_{\mathrm{B} 22}$ & 45 & 132 & 177 & 534.0 \\
$(+00)_{\mathrm{B} 22}$ & 10 & 0 & 10 & 0.0 \\
$(0--)_{\mathrm{B} 22}$ & 131 & 344 & 475 & 2089.6 \\
$(0-+)_{\mathrm{B} 22}$ & 0 & 0 & 0 & 0.0 \\
$(0-0)_{\mathrm{B} 22}$ & 15 & 0 & 15 & 0.0 \\
$(0+-)_{\mathrm{B} 22}$ & 48 & 172 & 220 & 738.4 \\
$(0++)_{\mathrm{B} 22}$ & 0 & 0 & 0 & 0.0 \\
$(0+0)_{\mathrm{B} 22}$ & 1 & 0 & 1 & 0.0 \\
$(00-)_{\mathrm{B} 22}$ & 179 & 0 & 179 & 0.0 \\
$(0 \quad 0+)_{\mathrm{B} 22}$ & 3 & 0 & 3 & 0.0 \\
$(000)_{\mathrm{B} 22}$ & 385 & 204 & 589 & 3178.0 \\
\hline Total & 101 & 1604 & 2705 & 8454.0 \\
\hline
\end{tabular}

Table 4 - Summary of QTC-B22 relations with their cumulative instant, interval, and total frequencies, and duration for $\mathbf{5 0 3}$ car pairs.

For the next group, we find the following correspondences for interval occurrences and durations:

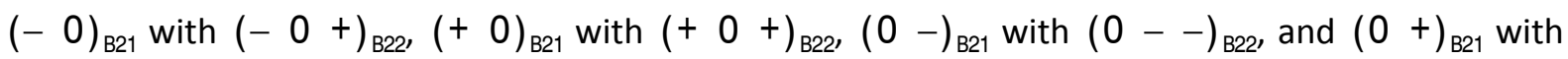
$(0--)_{\text {B22. }}$. Hence, the car that is certainly moving always has the highest speed. Presumably, this means that in most, if not all, of the cases the other car is standing still.

The group associated with cars that follow each other, has no instantaneous occurrences in B22 where the relative speed symbol is ' - ' or ' + ', as could be expected from restriction 2 (section 3.3) since those relations have no ' 0 ' symbols. 
Finally, the two remaining relations $(---)_{\mathrm{B} 22}$ and $(++-)_{\mathrm{B} 22}$ belong to the least represented groups, associated with convergence and divergence patterns. Due to their rare occurrence, four base relations are not represented in these groups.

\subsection{Squash rally}

In this case study, we analyse the relation of two squash opponents in a championship duel in QTCC22. Therefore, we employ the public standard CVBase'06 dataset (Pers, Bon \& Vuckovic 2006). In this dataset, the trajectories of two squash players were sampled from video frames taken at regular time steps of 0.04 seconds, by automatic computer vision based tracking under field expert supervision. The resulting trajectories were smoothed by a Gaussian kernel and have a positional RMS error of about $0.3 \mathrm{~m}$.

Since there is only one pair of players, there is no need for a cumulative summary table as in section 4.1. To simplify the discussion, we will consider the QTC-C22 relations between both players during a rally lasting $37 \mathrm{~s}$. For a complete chronological sequence of QTC-C22 relations during this rally, we refer to the Appendix B.

As stated in section 3.3, QTCAnalyst has the ability to compute relation patterns, i.e. chains of two or more subsequent relations. Let us now consider some simple permutable patterns. With simple patterns, we mean patterns that do not contain a repetition of subpatterns of a lower $\operatorname{order}^{2}$ (i.e. complex patterns). A permutable pattern represents a pattern and all permutations of it. For example, the patterns $a \rightarrow b \rightarrow c, b \rightarrow c \rightarrow a$, or $c \rightarrow a \rightarrow b$ all represent the same permutable pattern. Since the smallest order is two, patterns of order two and three are by definition simple patterns.

For the opponents in the rally, a total of 196 simple permutable QTC-C22 patterns of order four have been found, and they are distributed non uniformly both in terms of frequency and duration. 124 of them occur just once, i.e. only 72 patterns have at least one repetition. Figure 6 presents a graph of the frequency and the total duration for the 24 patterns with the longest total duration. All remaining patterns have a duration of less than $1.5 \mathrm{~s}$, and a frequency of eight or less. The graph shows that the three most frequent patterns also have the highest durations. Two patterns prevail: $(-+--)_{\mathrm{C} 22} \rightarrow(-0--)_{\mathrm{C} 22} \rightarrow(----)_{\mathrm{C} 22} \rightarrow(-0--)_{\mathrm{C} 22}$ with 40 occurrences over $7.8 \mathrm{~s}$, and $(+---)_{\mathrm{C} 22} \rightarrow(0---)_{\mathrm{C} 22} \rightarrow(----)_{\mathrm{C} 22} \rightarrow(0---)_{\mathrm{C} 22}$ with 20 occurrences over $8.9 \mathrm{~s}$. Interestingly, they are each other's converse pattern, and hence we learn that a prevailing movement behaviour during the rally, is that one player (follower) is following its opponent (leader) until the leader temporarily changes its moving direction towards the follower. Both players thereby continuously remain moving to the left of each other (i.e. to the left of the reference line connecting them). An alternation of both patterns may occur whenever the opponents are alternatively running in clockwise cycles around each other. In squash, this behaviour may arise whenever both players are alternating a forward move to play the ball with a backward move to let the opponent play, taking account of the interference rule. ${ }^{3}$

\footnotetext{
${ }^{2}$ The number of relations a pattern consists of.

${ }^{3}$ According to the official rules of squash (World Squash Federation 2009), "to avoid interference, the opponent must try to provide the player with unobstructed direct access to the ball, a fair view of the ball, space to complete a swing at the ball and freedom to play the ball directly to any part of the front wall".
} 
Note that both patterns have $(----)_{\mathrm{C22}}$ in common: the relation with the longest overall duration and second most occurrences (see Appendix B). Taking into account that the patterns are permutable, it follows that they might overlap with each other.

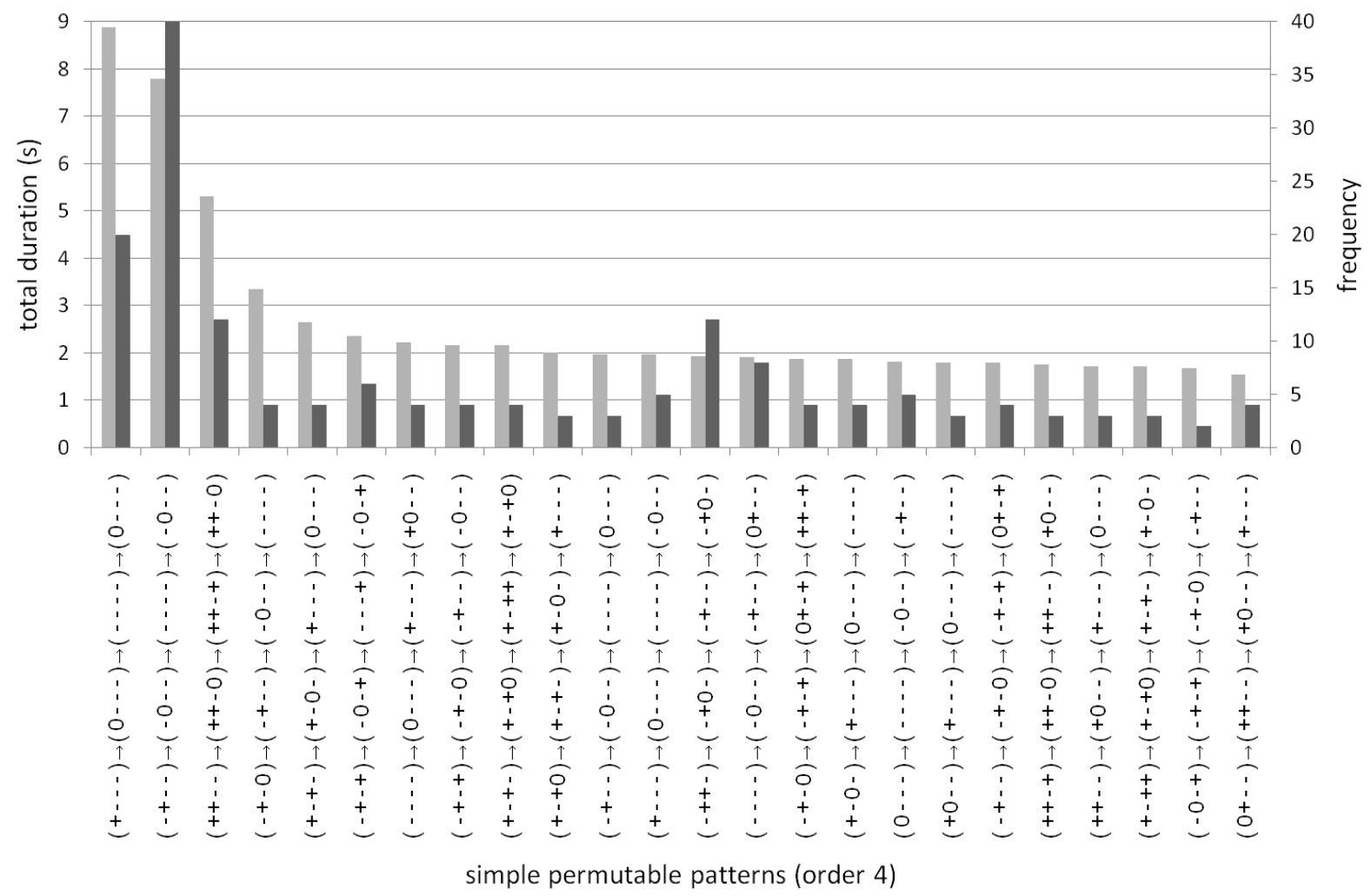

Figure 6 - Duration (gray bars) and frequency (black bars) for $\mathbf{2 4}$ fourth order simple permutable patterns in QTC-C22.

\section{Discussion}

This chapter has addressed the implementation of a QTC-based information system: we proposed a conceptual model, set up a prototype system, and demonstrated its applicability by two case studies. In what follows, we will point out some strengths and weaknesses of our approach.

(a) Although this chapter confines itself to an implementation for $\mathrm{QTC}_{B}$ and $\mathrm{QTC}_{C}$, the proposed conceptual model is based on the principles of continuity, and of jointly exhaustiveness and pairwise disjointness (JEPD), and is therefore generic for all QTC calculi. QTCAnalyst is not pretended to be an end-user information system. It is a use case independent prototype that may constitute the core of such a system, but would need enhanced processing capabilities and automatic interpretation mechanisms in order to become a fully fledged application. To illustrate the generality of this approach, we chose two completely different use cases in section 4.

(b) QTCAnalyst provides unambiguous and consistent results. Although there are no explicit implementations of conceptual neighbourhood diagrams (CNDs), QTCAnalyst results (e.g. Table 3, Table 4, and Figure 6) are consistent with conceptual neighbourhoodness, since the data respected the assumptions of continuity which conceptual neighbourhoodness captures, and no 
gaps in recording occurred. Explicit use of conceptual neighbourhoods could be useful in the case of incomplete data. For instance, if at some instants the position of some MPOs is not known, possible relations may be inferable through the assumptions of continuity as encoded in the CNDs. Alternatively, interpolation on the actual positions could of course be used.

(c) While being a key and much studied operation in qualitative reasoning generally, the composition of relations is not supported by QTCAnalyst. The starting point for QTCAnalyst is trajectory data, and hence, in order to determine the relations $R(a, b)$ and $R(b, c)$, the trajectory data of all objects $a, b$, and $c$ is required. Consequently, rather than applying the composition of $R(a, b)$ and $R(b, c)$, a direct computation of $R(a, c)$ is more convenient, more efficient, and often more precise. We do not ignore the usefulness of composition, and it provides another method, in addition to the use of conceptual neighbourhoods mentioned in (b) above to inferring missing data; however, composition has not been the focus in this work.

(d) Although Assumptions 1 and 2 can be used to interpolate MPO trajectories, some open problems still remain in the MPO modelling domain. According to Laube, Imfeld and Weibel (2005) these include the presence of uncertain and/or missing fixes as well as granularity related issues. The granularity issue is worth discussing. Consider for instance case A in Figure 7, where

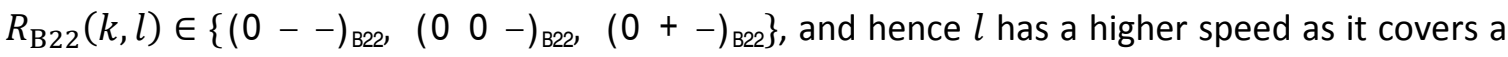
longer distance in reality (Figure 7a). However, due to a sampling satisfying Assumptions 1-3, this case may be represented as in Figure $7 \mathrm{~b}$, where $R_{\mathrm{B} 22}(k, l)=\left(\begin{array}{lll}0 & 0 & 0\end{array}\right)_{\mathrm{B} 22 .}$. Another example is case $\mathrm{B}$, where $k$ is circling around a stationary $l$, and thus $R_{\mathrm{B} 21}(k, l)=\left(\begin{array}{ll}0 & 0\end{array}\right)_{\mathrm{B} 21}$ (Figure 7c). However, such a circular trajectory can never be represented by straight segments, and thus $R_{\mathrm{B} 21}(k, l)$ will never equal $\left(\begin{array}{ll}0 & 0\end{array}\right)_{\mathrm{B} 21}$ over a time interval in Figure $7 \mathrm{~d}$. In both cases, wrong relations are obtained due to the sampling of fixes and the application of Assumptions 1-3. Moreover, they illustrate how relative speed as well as relative direction may be influenced by a sampled representation. Note that both cases deal with pertaining stable relations, i.e. relations with a ' 0 ' symbol holding over a time interval: in case $A$, ' $O$ ' erroneously arises, whereas in case $B$, ' $O$ ' is erroneously missed. Yet, there is another important difference: in case $A$, the error could be avoided by sufficient sampling granularity, whereas this does not apply for case $B$. In order to obtain the desired stable relations, for situations such as case $B$, there are several possible solutions. A reasonable one could be to introduce spatiotemporal limits or thresholds, where stable relations occur whenever movements (such as relative speeds, or relative directions) remain within these presumed thresholds. Depending upon the field of application, suitable thresholds may be based on the precision or accuracy of the trajectory data, the user-intended analysis granularity, the limits of the object's (human, animal, robot, etc.) perception, etc. This approach will also increase the chance of stable relations actually occurring, where otherwise the usefulness of these so called borderline cases is sometimes questioned (Gottfried 2008).

(e) While Assumptions 1-2 are applicable to every set of fixes, Assumption 3 is the only one restricting the data collection method. In addition, this assumption may sometimes be unrealistic, for instance when multiple unsynchronised sensors are used or in case of missing fixes. However, as mentioned earlier, one can easily obtain concurrency by applying first the Assumptions 1-2, and then resample the resulting trajectory in order to fulfil Assumption 3. 
case $\mathrm{A}$

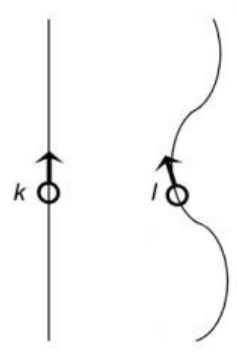

(a)

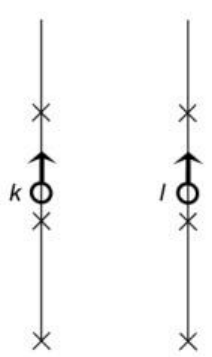

(b)

case B

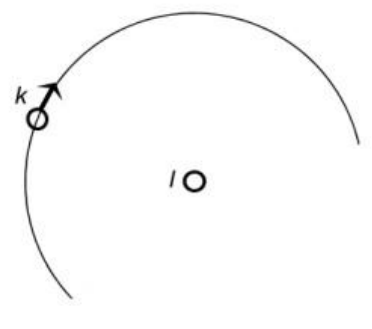

(c)

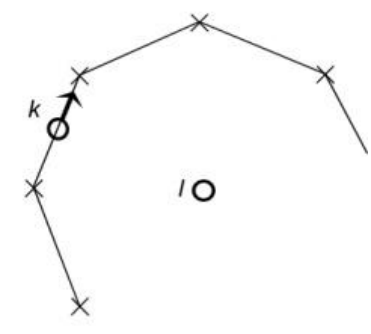

(d)

Figure 7 - Trajectories of two objects $k$ and / during a time interval for two situations (A and B) according to two representations: realistic representation (a), (c); representation satisfying Assumptions 1-3 (b), (d). Crosses represent fixes.

(f) Throughout this chapter, the usual, absolute notion of time has been employed. However, since QTC considers relative relationships, a relative time notion could be useful in some cases, e.g. to compare movements with different time spans.

(g) In an applied setting, a user's interest in QTC relations will be limited to those objects that interact with each other. The assessment of interacting objects, i.e. the issue of determining exactly which objects are interacting with each other, has been indicated as an open problem (Andrienko et al. 2008). Hence, the advances made on that issue might improve further implementations.

(h) The case studies in section 4 have shown that QTCAnalyst might be a useful tool for the analysis of trajectory data within diverse contexts, such as traffic monitoring or sports performance analysis. It is commonly accepted that qualitative and quantitative formalisms should complement each other. This idea also underlies the results in section 4, were we analysed the quantitative properties of qualitative relations or patterns.

\section{Conclusions and outlook}

The contribution of this chapter is threefold:

- A generic conceptual model for the analysis of moving point objects through a Qualitative Trajectory Calculus was introduced.

- An implementation methodology was proposed for the $\mathrm{QTC}_{\mathrm{B}}$ and $\mathrm{QTC}_{\mathrm{C}}$ calculi and a prototype was developed (QTCAnalyst).

- The applicability for a QTC-based information system was highlighted in two case studies.

In future work, we intend to extend QTCAnalyst to other QTC calculi, especially QTC $_{\mathrm{N}}$. Since QTC abstracts from the actual moving objects, its implementation is not our first priority, though there may be promising applications, e.g. in the field of trajectory similarity measurement.

Also, a more advanced implementation may call for an explicit representation of conceptual neighbourhood diagrams as well as composition tables. 
As mentioned in the discussion, a fully fledged information system should implement advanced mechanisms for post-processing and automatically interpreting raw QTC relations and/or patterns. Moreover, its input modalities should go beyond conventional trajectory data: whereas the intuitiveness and suitability for human decision making is a key motivation for qualitative reasoning systems, possibilities for user-interactive and intuition-based data input, such as query-by-sketch (Egenhofer 1996) should be considered. On the other hand, these extensions would in turn benefit from increased output capabilities such as advanced visualisation and communication means, e.g. animations.

\section{References}

Allen, J. F. (1983) Maintaining knowledge about temporal intervals. Communications of the ACM, 26, 11, 832-843.

Andrienko, N., Andrienko, G., Wachowicz, M. \& Orellana, D. (2008) Uncovering interactions between moving objects. In Proceedings of the 5th International Conference on Geographic Information Science, eds. T. J. Cova, H. J. Miller, K. Beard, A. U. Frank \& M. F. Goodchild, Park City, Utah, 16-26.

Bogaert, P. (2008) A Qualitative Calculus for Moving Point Objects Constrained by Networks, Doctoral Dissertation, Ghent: Ghent University, 155.

Brakatsoulas, S., Pfoser, D., Salas, R. \& Wenk, C. (2005) On map-matching vehicle tracking data. In Proceedings of the 31st International Conference on Very Large Data Bases, Trondheim, Norway: VLDB Endowment, 853-864.

Brakatsoulas, S., Pfoser, D. \& Tryfona, N. (2004) Modeling, storing, and mining moving object databases. In Proceedings of the International Database Engineering and Applications Symposium, IEEE Computer Society, 68-77.

Brunk, B. \& Davis, B. (2002) SDAT enterprise: Application of geospatial network services for collaborative airspace analysis. In CADD/GIS Symposium Proceedings, San Antonio.

DeCesare, N. J., Squires, J. R. \& Kolbe, J. A. (2005) Effect of forest canopy on GPS-based movement data. Wildlife Society Bulletin, 33, 3, 935-941.

Delafontaine, M., Van de Weghe, N., Bogaert, P. \& De Maeyer, P. (2008) Qualitative relations between moving objects in a network changing its topological relations. Information Sciences, 178, 8, 1997-2006.

Eagle, N. \& Pentland, A. (2006) Reality mining: sensing complex social systems. Personal and Ubiquitous Computing, 10, 4, 255-268.

Egenhofer, M. J. (1996) Spatial-Query-by-Sketch. In Proceedings of the 1996 IEEE Symposium on Visual Languages, 60-67.

Egenhofer, M. J. \& Mark, D. (1995) Naive geography. In Spatial Information Theory: A Theoretical Basis for GIS, eds. A. U. Frank \& W. Kuhn, Berlin / Heidelberg: Springer-Verlag, 1-15.

El-Geresy, B. A. \& Abdelmoty, A. I. (2004) SPARQS: a qualitative spatial reasoning engine. KnowledgeBased Systems, 17, 2-4, 89-102.

Frank, A., U. (1991) Qualitative spatial reasoning about cardinal directions. In Proceedings of Autocarto 10, American Congress on Surveying and Mapping, eds. D. Mark \& D. White, Baltimore, Maryland, 148-167.

Freksa, C. (1992a) Temporal reasoning based on semi-intervals. Artificial Intelligence, 54, 199-127. 
Freksa, C. (1992b) Using orientation information for qualitative spatial reasoning. In Theories and Methods of Spatio-Temporal Reasoning in Geographic Space, eds. A. U. Frank, I. Campari \& U. Formentini, Pisa, Italy: Springer-Verlag, 162-178.

Gagliardo, A., loale, P., Savini, M., Lipp, H. P. \& Dell'Omo, G. (2007) Finding home: the final step of the pigeons' homing process studied with a GPS data logger. Journal of Experimental Biology, 210, 7, 1132-1138.

Galton, A. (2001) Dominance diagrams: a tool for qualitative reasoning about continuous systems. Fundamenta Informaticae, 46, 1-2, 55-70.

Gau, R. J., Mulders, R., Ciarniello, L. J., Heard, D. C., Chetkiewicz, C. L. B., Boyce, M., Munro, R., Stenhouse, G., Chruszcz, B., Gibeau, M. L., Milakovic, B. \& Parker, K. L. (2004) Uncontrolled field performance of televilt GPS-Simplex (TM) collars on grizzly bears in western and northern Canada. Wildlife Society Bulletin, 32, 3, 693-701.

Giannotti, F. \& Pedreschi, D. (2008) Mobility, Data Mining and Privacy: Geographic Knowledge Discovery, Springer, 410.

Gottfried, B. (2008) Representing short-term observations of moving objects by a simple visual language. Journal of Visual Languages and Computing, 19, 3, 321-342.

Grace, J. (2000) GPS guidance system increases projectile accuracy. IEEE Aerospace and Electronic Systems Magazine, 15, 6, 15-17.

Gudmundsson, J., van Kreveld, M. \& Speckmann, B. (2007) Efficient detection of patterns in 2D trajectories of moving points. Geoinformatica, 11, 195-215.

Güting, R., Hartmut, de Almeida, V., Teixeira \& Ding, Z. (2006) Modeling and querying moving objects in networks. The International Journal on Very Large Data Bases, 15, 2, 165-190.

Hornsby, K. \& Egenhofer, M. J. (2002) Modeling moving objects over multiple granularities. Annals of Mathematics and Artificial Intelligence, 36, 1-2, 177-194.

Hvidberg, M. (2006) Tracking human exposure to ultrafine particles in Copenhagen using GPS. Epidemiology, 17, 6, S38-S38.

Kritzler, M., Raubal, M. \& Kruger, A. (2007) A GIS framework for spatio-temporal analysis and visualization of laboratory mice tracking data. Transactions in GIS, 11, 5, 765-782.

Laube, P., Dennis, T., Forer, P. \& Walker, M. (2007) Movement beyond the snapshot - Dynamic analysis of geospatial lifelines. Computers, Environment and Urban Systems, 31, 5, 481-501.

Laube, P., Imfeld, S. \& Weibel, R. (2005) Discovering relative motion patterns in groups of moving point objects. International Journal of Geographical Information Science, 19, 6, 639 - 668.

Laube, P., van Kreveld, M. \& Imfeld, S. (2005) Finding REMO - Detecting relative motion patterns in geospatial lifelines. In Developments in Spatial Data Handling, ed. P. Fisher, Berlin: SpringerVerlag, 201-215.

Longley, P. A., Goodchild, M. F., Maquire, D. J. \& Rhind, D. W. (2005) Geographic Information Systems and Science. John Wiley \& Sons, 519.

Mark, D. M. (1998) Geospatial lifelines. In Integrating Spatial and Temporal Databases. Dagstuhl Seminars.

Michael, K., McNamee, A., Michael, M. G. \& Tootell, H. (2006) Location-based intelligence - modeling behavior in humans using GPS. In IEEE International Symposium on Technology and Society (ISTAS 2006) 97-104.

Monferrer, T. E. \& Lobo, F. T. (2002) Qualitative velocity. In Proceedings of the 5th Catalonian Conference on Al: Topics in Artificial Intelligence, Springer-Verlag, 29-39. 
Moratz, R., Dylla, F. \& Frommberger, L. (2005) A relative orientation algebra with adjustable granularity. In Proceedings of the Workshop on Agents in Real-Time and Dynamic Environments (IJCAI 05), Edinburgh, Scotland.

Nielsen, T., S. \& Hovgesen, H., H. (2004) GPS in pedestrian and spatial behaviour surveys. In Cities for People, Proceedings of the 5th International Conference on Walking in the 21st Century, 13. Copenhagen, Denmark.

Orlando, S., Orsini, R., Raffaeta, A., Roncato, A. \& Silverstri, C. (2007) Trajectory data warehouses: design and implementation issues. Journal of Computing Science and Engineering, 1, 2, 211232.

Pers, J., Bon, M. \& Vuckovic, G. (2006) CVBASE '06 Dataset, available online: http://vision.fe.unilj.si/cvbase06/dataset.html.

Randell, D. A., Cui, Z. \& Cohn, A. G. (1992) A spatial logic based on regions and connection. In Proceedings of the 3rd International Conference on Principles of Knowledge Representation and Reasoning, eds. B. Nebel, C. Rich \& W. Swartout, 165-176.

Renz, J. \& Li, J. J. (2008) Automated complexity proofs for qualitative spatial and temporal calculi. In Proceedings of the 11th International Conference on Principles of Knowledge Representation and Reasoning (KR'08), Sydney, Australia, 715-723.

Renz, J., Rauh, R. \& Knauff, M. (2000) Towards cognitive adequacy of topological spatial relations. In Spatial Cognition II, LNCS 1849, 184-197.

Spaccapietra, S., Parent, C., Damiani, M. L., de Macedo, J. A., Portoa, F. \& Vangenot, C. (2008) A conceptual view on trajectories. Data \& Knowledge Engineering, 65, 1, 126-146.

Turchin, P. (1998) Quantitative Analysis of Movement: Measuring and Modeling Population Redistribution in Animals and Plants. Sunderland, MA: Sinauer, 396.

Van de Weghe, N. (2004) Representing and Reasoning about Moving Objects: A Qualitative Approach, Doctoral Dissertation, Ghent: Ghent University, 268.

Van de Weghe, N., Cohn, A. G., De Maeyer, P. \& Witlox, F. (2005a) Representing moving objects in computer-based expert systems: the overtake event example. Expert Systems With Applications, 29, 4, 977-983.

Van de Weghe, N., Cohn, A. G., De Tré, G. \& De Maeyer, P. (2006) A Qualitative Trajectory Calculus as a basis for representing moving objects in geographical information systems. Control and Cybernetics, 35, 1, 97-119.

Van de Weghe, N., De Tré, G., Kuijpers, B. \& De Maeyer, P. (2005b) The double-cross and the generalization concept as a basis for representing and comparing shapes of polylines. In Proceedings of the 1st International Workshop on Semantic-based Geographical Information Systems (SeBGIS'05), Agia Napa, Cyprus.

Van de Weghe, N., Kuijpers, B., Bogaert, P. \& De Maeyer, P. (2005c) A Qualitative Trajectory Calculus and the composition of its relations. In Proceedings of the 1st International Conference on Geospatial Semantics (GeoS), eds. A. M. Rodríguez, I. F. Cruz, M. J. Egenhofer \& S. Levashkin, Springer-Verlag, 60-76.

Vilain, M. \& Kautz, H. (1986) Constraint propagation algorithms for temporal reasoning. In AAAI-86 Proceedings, 377-382.

Wallgrün, J. O., Frommberger, L., Wolter, D., Dylla, F. \& Freksa, C. (2007) Qualitative spatial representation and reasoning in the SparQ-toolbox. In Spatial Cognition V: Reasoning, Action, Interaction, eds. T. Barkowsky, M. Knauff, G. Ligozat \& D. R. Montello, 39-58. 
Wang, L., Hu, W. \& Tan, T. (2003) Recent developments in human motion analysis. Pattern Recognition, 36, 3, 585-601.

Wentz, E. A., Campbell, A. F. \& Houston, R. (2003) A comparison of two methods to create tracks of moving objects: linear weighted distance and constrained random walk. International Journal of Geographical Information Science, 17, 7, 623-645.

World Squash Federation (2009) World Squash Singles Rules, World Squash Federation, 33.

Yasuda, T. \& Arai, N. (2005) Fine-scale tracking of marine turtles using GPS-argos PTTs. Zoological Science, 22, 5, 547-553.

Yu, B. \& Kim, S. (2006) Interpolating and using most likely trajectories in moving-objects databases. In Database and Expert Systems Applications, LNCS 4080, 718-727.

Yu, B., Kim, S. H., Bailey, T. \& Gamboa, R. (2004) Curve-Based representation of moving object trajectories. In Proceedings of the International Database Engineering and Applications Symposium, IEEE Computer Society, 419-425.

Zimmermann, K. \& Freksa, C. (1996) Qualitative spatial reasoning using orientation, distance, and path knowledge. Applied Intelligence, 6, 1, 49-58. 


\section{Appendix A}

Consider two MPOs $k$ and $l$ with $k^{t}, l^{t}, \overrightarrow{v_{k}^{t}}, \overrightarrow{v_{l}^{t}}, k^{t} l^{t}, \overrightarrow{k^{t} l^{t}}, \overrightarrow{l^{t} k^{t}}, \alpha^{t}, \beta^{t}, \gamma^{t}, \delta^{t}$ as defined in section 2.2.

Let us consider a two-dimensional space, so that $k^{t}, l^{t} \in \mathbb{R}^{2}$. Let $t_{1}, t_{2}$ be the time instants of two consecutive fixes, so that:

$$
\begin{aligned}
& k^{t 1}=\left(x_{k}^{t_{1}}, y_{k}^{t_{1}}\right), k^{t 2}=\left(x_{k}^{t_{2}}, y_{k}^{t_{2}}\right), \\
& l^{t 1}=\left(x_{l}^{t_{1}}, y_{l}^{t_{1}}\right), l^{t 2}=\left(x_{l}^{t_{2}}, y_{l}^{t_{2}}\right) .
\end{aligned}
$$

Then, due to Assumptions 1-3:

$$
\begin{array}{ll}
\forall t \in] t_{1}, t_{2}[: & k^{t}=\left(x_{k}^{t}, y_{k}^{t}\right) \\
& l^{t}=\left(x_{l}^{t}, y_{l}^{t}\right)
\end{array}
$$

with

$$
\begin{aligned}
& x_{k}^{t}=x_{k}^{t_{1}}+\left(x_{k}^{t_{2}}-x_{k}^{t_{1}}\right)\left(t-t_{1}\right) \\
& y_{k}^{t}=y_{k}^{t_{1}}+\left(y_{k}^{t_{2}}-y_{k}^{t_{1}}\right)\left(t-t_{1}\right) \\
& x_{l}^{t}=x_{l}^{t_{1}}+\left(x_{l}^{t_{2}}-x_{l}^{t_{1}}\right)\left(t-t_{1}\right) \\
& y_{l}^{t}=y_{l}^{t_{1}}+\left(y_{l}^{t_{2}}-y_{l}^{t_{1}}\right)\left(t-t_{1}\right)
\end{aligned}
$$

For $\overrightarrow{v_{k}^{t}}, \overrightarrow{v_{l}^{t}}, k^{t} l^{t}, \overrightarrow{k^{t} l^{t}}, \overrightarrow{l^{t} k^{t}}, \alpha^{t}, \beta^{t}, \gamma^{t}, \delta^{t}$ as functions of $t$, it follows from (5.13-5.16):

$$
\begin{aligned}
& \operatorname{deg}\left(\overrightarrow{v_{k}^{t}}\right)=0 \\
& \operatorname{deg}\left(\overrightarrow{v_{l}^{t}}\right)=0 \\
& \operatorname{deg}\left(k^{t} l^{t}\right)=2 \\
& \operatorname{deg}\left(\overrightarrow{k^{t} l^{t}}\right)=1 \\
& \operatorname{deg}\left(\overrightarrow{l^{t} k^{t}}\right)=1
\end{aligned}
$$

Hence, due to (5.17-5.21):

$$
\begin{aligned}
& \operatorname{deg}(\alpha)=1 \\
& \operatorname{deg}(\beta)=1 \\
& \operatorname{deg}(\gamma)=2 \\
& \operatorname{deg}(\delta)=2
\end{aligned}
$$


Appendix B

\begin{tabular}{|c|c|c|c|c|c|}
\hline Relation & Time & Duration & $(+0--)_{\mathrm{C} 21}$ & 34.78 & \\
\hline unknown & 28.00 & & $(++--)_{\mathrm{C} 21}$ & & 0.02 \\
\hline$(-+-+)_{C 21}$ & & 0.04 & $(++0-)_{\mathrm{C} 21}$ & 34.80 & \\
\hline$(-0-+)_{\mathrm{C} 21}$ & 28.04 & & $(+++-)_{C 21}$ & & 0.76 \\
\hline$(---+)_{\mathrm{C}_{21}}$ & & 0.16 & $(+0+-)_{\mathrm{C} 21}$ & 35.56 & \\
\hline$(-0-+)_{c 21}$ & 28.20 & & $(+-+-)_{C_{21}}$ & & 0.08 \\
\hline$(-+-+)_{\mathrm{C}_{21}}$ & & 0.20 & $(0-+0)_{\mathrm{C} 21}$ & 35.64 & \\
\hline$(-0-+)_{C 21}$ & 28.40 & & $(--++)_{\mathrm{C} 21}$ & & 0.04 \\
\hline$(-\ldots+)_{c 21}$ & & 0.12 & $(-0+)_{c 21}$ & 35.68 & \\
\hline$(-0-+)_{C 21}$ & 28.52 & & $(--++)_{\mathrm{C} 21}$ & & 0.04 \\
\hline$(-+-+)_{\mathrm{C} 21}$ & & 0.68 & $(-0-+)_{C 21}$ & 35.72 & \\
\hline$(-+-0)_{\mathrm{C}_{21}}$ & 29.20 & & $(-+-+)_{C 21}$ & & 0.04 \\
\hline$(-+--)_{C 21}$ & & 0.80 & $(-+-0)_{\mathrm{C} 21}$ & 35.76 & \\
\hline$\left(\begin{array}{lll}-0 & - & -\end{array}\right)_{\mathrm{C} 21}$ & 30.00 & & $(-+--)_{C 21}$ & & 0.16 \\
\hline$(----)_{\mathrm{C} 21}$ & & 0.20 & $(-0---)_{c 21}$ & 35.92 & \\
\hline$(-0--)_{c 21}$ & 30.20 & & $(----)_{\mathrm{C} 21}$ & & 0.59 \\
\hline$(-+--)_{\mathrm{C} 21}$ & & 0.08 & $(0---)_{\mathrm{C} 21}$ & 36.51 & \\
\hline$(-0--)_{C 21}$ & 30.28 & & $(+-\quad-\quad-)_{\mathrm{C} 21}$ & & 0.02 \\
\hline$(-\quad--)_{C_{21}}$ & & 0.16 & $(+0--)_{C_{21}}$ & 36.53 & \\
\hline$\left(\begin{array}{lll}- & - & -\end{array}\right)_{\mathrm{C} 21}$ & 30.44 & & $(++--)_{C 21}$ & & 0.31 \\
\hline$(--+-)_{c 21}$ & & 0.12 & $(++-0)_{\mathrm{C} 21}$ & 36.84 & \\
\hline$(-0+-)_{C 21}$ & 30.56 & & $(++-+)_{\mathrm{C}_{21}}$ & & 0.12 \\
\hline$(-++-)_{C 21}$ & & 0.12 & $(+0-+)_{\mathrm{C} 21}$ & 36.96 & \\
\hline$(-0+-)_{c 21}$ & 30.68 & & $(+--+)_{\mathrm{c} 21}$ & & 0.08 \\
\hline$(--+-)_{\mathrm{C}_{21}}$ & & 0.24 & $(+--0)_{\mathrm{C} 21}$ & 37.04 & \\
\hline$(--+0)_{\mathrm{C} 21}$ & 30.92 & & $(+-\quad-\quad-)_{\mathrm{C} 21}$ & & 0.24 \\
\hline$(--++)_{\mathrm{C} 21}$ & & 0.28 & $(0---)_{\mathrm{c} 21}$ & 37.28 & \\
\hline$(0-++)_{\mathrm{C} 21}$ & 31.20 & & $(----)_{\mathrm{C} 21}$ & & 0.04 \\
\hline$(+-++)_{\mathrm{C}_{21}}$ & & 0.40 & $\left(\begin{array}{lll}- & -0 & -\end{array}\right)_{\mathrm{C} 21}$ & 37.32 & \\
\hline$(+-0+)_{C 21}$ & 31.60 & & $(--+-)_{\mathrm{C} 21}$ & & 0.12 \\
\hline$(+--+)_{\mathrm{C}_{21}}$ & & 0.12 & $(-0+-)_{\mathrm{C}_{21}}$ & 37.44 & \\
\hline$(+0-+)_{C 21}$ & 31.72 & & $(-++-)_{\mathrm{C} 21}$ & & 0.27 \\
\hline$(++-+)_{\mathrm{c} 21}$ & & 0.24 & $(-+0-)_{C_{21}}$ & 37.71 & \\
\hline$(0+0+)_{\mathrm{C} 21}$ & 31.96 & & $(-+--)_{\mathrm{C} 21}$ & & 0.01 \\
\hline$(-+++)_{C 21}$ & & 0.20 & $(-+0-)_{\mathrm{C} 21}$ & 37.72 & \\
\hline$(-++0)_{\mathrm{C} 21}$ & 32.16 & & $(-++-)_{\mathrm{C} 21}$ & & 0.02 \\
\hline$(-++-)_{C 21}$ & & 0.36 & $(-+0-)_{\mathrm{C} 21}$ & 37.74 & \\
\hline$(-+0-)_{c 21}$ & 32.52 & & $(-+--)_{\mathrm{C} 21}$ & & 0.02 \\
\hline$(-+--)_{c 21}$ & & 0.16 & 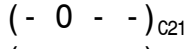 & 37.76 & \\
\hline$(-+-0)_{\mathrm{C} 21}$ & 32.68 & & $(-\quad-\quad-\quad)_{\mathrm{C} 21}$ & & 0.02 \\
\hline$(-+-+)_{C_{21}}$ & & 0.52 & $(-0--)_{\mathrm{C} 21}$ & 37.78 & \\
\hline$(0+-+)_{\mathrm{C} 21}$ & 33.20 & & $(-+--)_{C 21}$ & & 0.02 \\
\hline$(++-+)_{\mathrm{C}_{21}}$ & & 0.16 & $\left(\begin{array}{lll}-0 & - & -\end{array}\right)_{\mathrm{C} 21}$ & 37.80 & \\
\hline$(0+-0)_{\mathrm{C} 21}$ & 33.36 & & $(----)_{\mathrm{C} 21}$ & & 0.11 \\
\hline$(-+--)_{C 21}$ & & 0.03 & 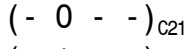 & 37.91 & \\
\hline$(0+--)_{\mathrm{C}_{21}}$ & 33.39 & & $(-+--)_{\mathrm{C} 21}$ & & 0.01 \\
\hline$(++--)_{\mathrm{C}_{21}}$ & & 0.01 & $(-0---)_{\mathrm{C} 21}$ & 37.92 & \\
\hline$(0+--)_{\mathrm{C} 21}$ & 33.40 & & $(----)_{\mathrm{C} 21}$ & & 0.01 \\
\hline$(-+--)_{\mathrm{C} 21}$ & & 0.28 & $(-0--)_{\mathrm{C} 21}$ & 37.93 & \\
\hline$(-0--)_{\mathrm{C} 21}$ & 33.68 & & $(-+--)_{\mathrm{C} 21}$ & & 0.09 \\
\hline$(----)_{\mathrm{C} 21}$ & & 0.24 & $(0+--)_{\mathrm{C} 21}$ & 38.02 & \\
\hline$(0---)_{c 21}$ & 33.92 & & $(++--)_{\mathrm{C} 21}$ & & 0.46 \\
\hline$(+-\quad--)_{\mathrm{C}_{21}}$ & & 0.16 & $(+0--)_{C_{21}}$ & 38.48 & \\
\hline$(0---)_{\mathrm{C} 21}$ & 34.08 & & $(+-\quad-\quad-)_{C 21}$ & & 0.12 \\
\hline$(-\quad-\quad-)_{C 21}$ & & 0.55 & $(0---)_{\mathrm{C} 21}$ & 38.60 & \\
\hline
\end{tabular}

( - - - - )

$(0---)_{\mathrm{C} 21}$

$(+--)_{\mathrm{C} 21}$

$(0 \text { - - - })_{\mathrm{C} 21}$

( - - - - ) c21

$(-0--)_{\mathrm{C} 21}$

$(-+--)_{\mathrm{C} 21}$

$(0+--)_{\text {c21 }}$

$(++--)_{\text {C21 }}$

$(0+-0)_{\text {C21 }}$

$(-+-+)_{\mathrm{C} 21}$

$(0+-+)_{\mathrm{C} 21}$

$(++-+)_{\mathrm{C} 21}$

$(+0-0)_{\mathrm{C} 21}$

$(+-)_{\mathrm{C} 21}$

$(+-\quad 0)_{\text {C21 }}$

$(+-+)_{\mathrm{C} 21}$

$(+-\quad 0)_{\mathrm{c} 21}$

$(+-)_{\mathrm{C} 21}$

$(+0--)_{\mathrm{C} 21}$

$(++--)_{\text {C21 }}$

$(+0--)_{\mathrm{c} 21}$

$(+---)_{\mathrm{C} 21}$

$(+-0-)_{\mathrm{c} 21}$

$(+-+-)_{\mathrm{C} 21}$

$(+0+-)_{\mathrm{c} 21}$

$(+++-)_{\mathrm{C} 21}$

$(+0+-)_{\mathrm{C} 21}$

$(+-+-)_{\mathrm{C} 21}$

$(+-+0)_{\mathrm{C} 21}$

$(+-++)_{\mathrm{C} 21}$

$(0-++)_{\mathrm{C} 21}$

$(--++)_{\mathrm{C} 21}$

$(-\quad 0+)_{\mathrm{c} 21}$

$(--\quad+)_{\mathrm{C} 21}$

$(--0)_{\mathrm{C} 21}$

( - - - - ) $)_{\text {C21 }}$

$(0---)_{\mathrm{C} 21}$

$(+-)_{\mathrm{C} 21}$

$(+0--)_{\mathrm{c} 21}$

$(++--)_{\mathrm{C} 21}$

$(+0--)_{\mathrm{C} 21}$

$(+--)_{\mathrm{C} 21}$

$(+0--)_{\mathrm{C} 21}$

$(++--)_{\mathrm{C} 21}$

$(0+--)_{\mathrm{C} 21}$

$(-+--)_{\mathrm{C} 21}$

$(0+--)_{\text {C21 }}$

$(++--)_{\mathrm{C} 21}$

$(+0--)_{\mathrm{c} 21}$

(+ - - - ) $)_{\mathrm{C} 21}$

$(+-\quad 0)_{\mathrm{C} 21}$

$(+-++)_{\mathrm{C} 21}$

$(+-\quad 0)_{\mathrm{C} 21}$

(+ - - - ) c21

$(+-0-)_{\mathrm{c} 21}$

39.20

39.32

0.12

0.28

39.60

0.24

39.84

0.00

39.84

0.01

39.85

0.39

40.24

0.04

40.28

40.32

0.04

0.04

40.36

0.36

40.72

0.08

40.80

0.12

40.92

0.12

41.04

0.09

41.13

0.55

41.68

0.08

41.76

0.20

41.96

0.16

42.12

0.28

42.40

0.00

42.40

0.00

42.40

0.04

42.44

0.31

42.75

0.05

42.80

0.12

42.92

0.40

43.32

0.08

$(+-+-)_{\mathrm{C} 21}$

43.40

0.56 


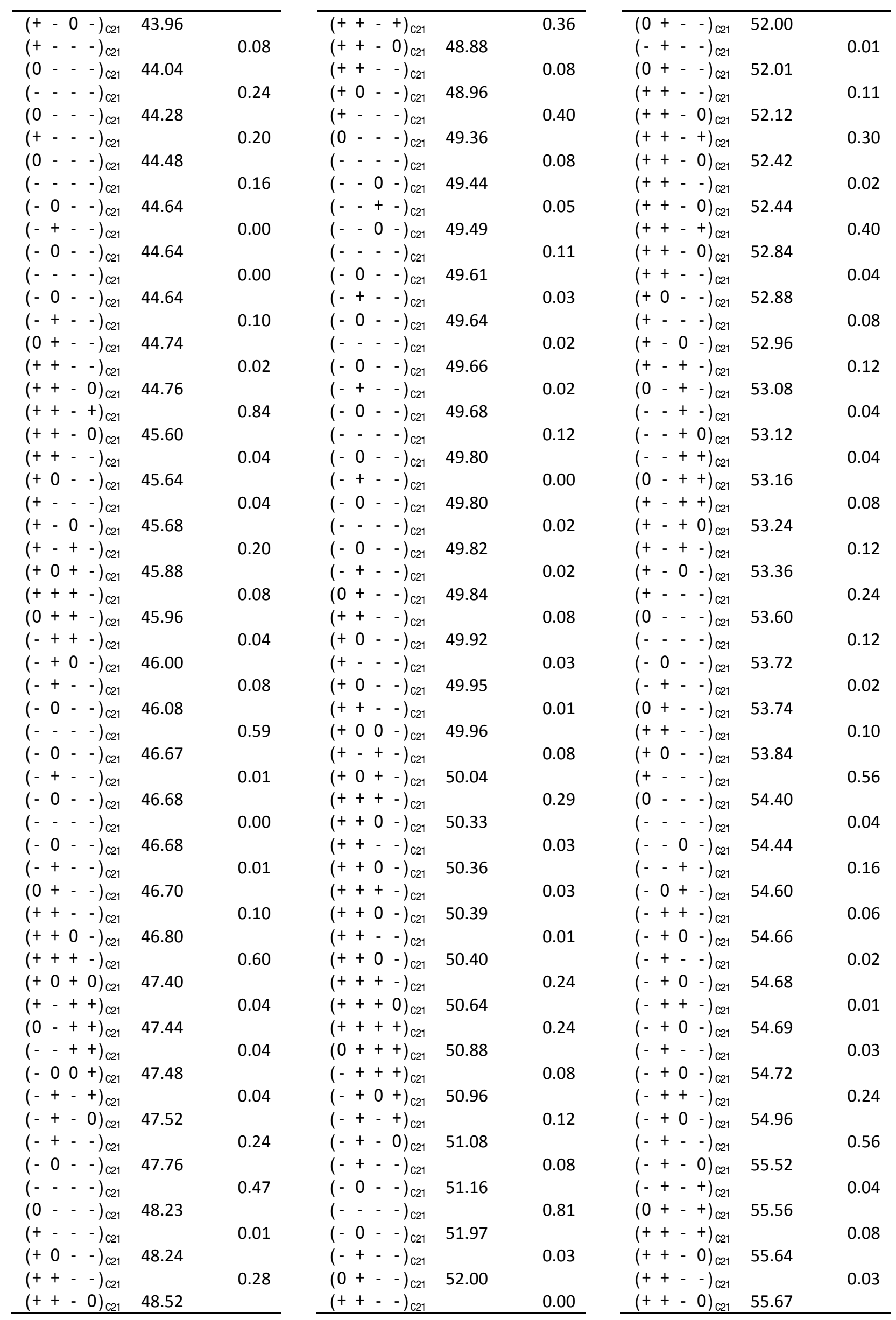




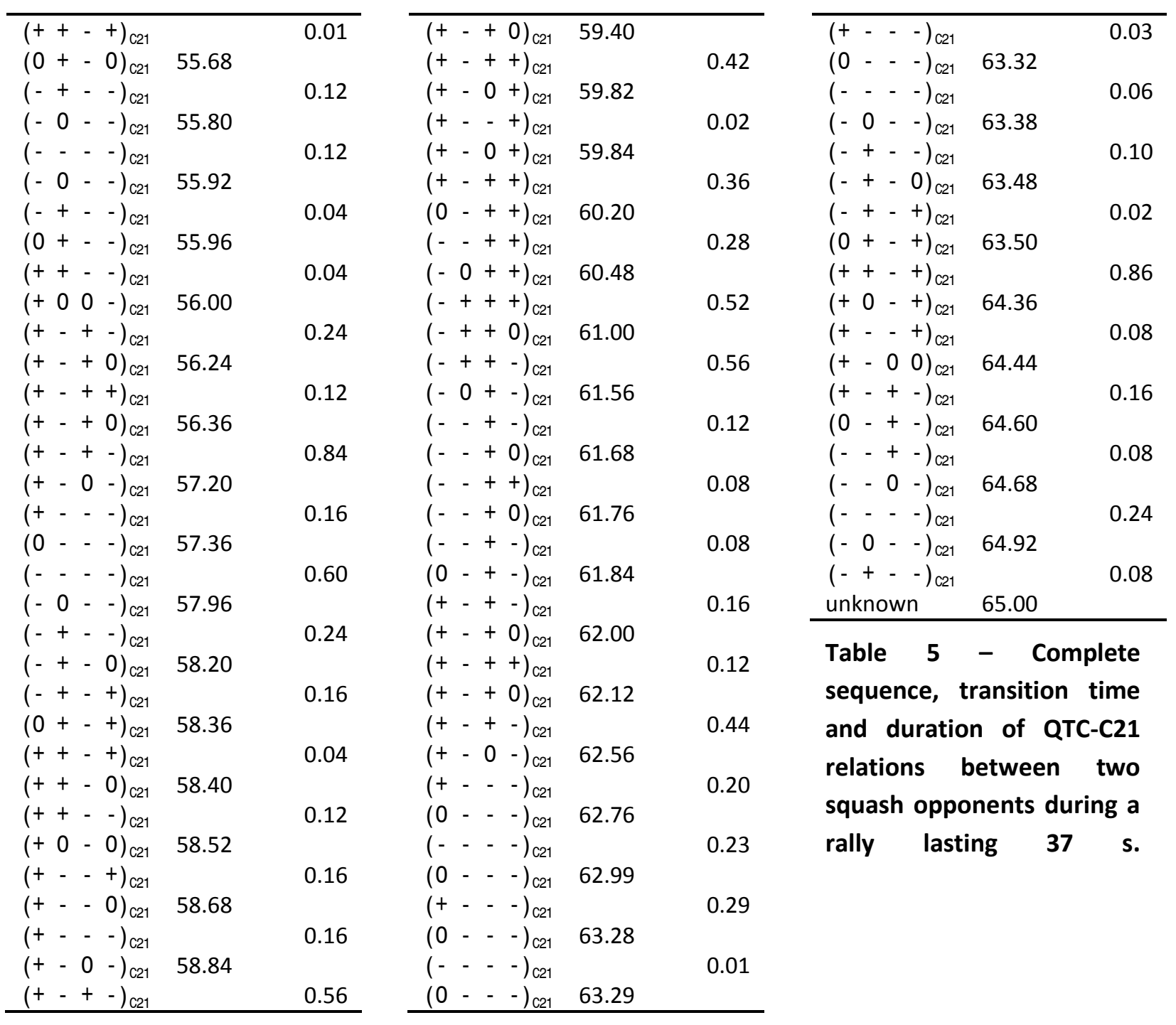

